\title{
Desempeño de los procedimientos laparoscópicos en ginecología: adquisición y mantenimiento de la idoneidad
}

\author{
Víctor Vázquez Zárate, Jorge Zepeda Zaragoza, Carlos Briones Landa, \\ Mariana Hernández Ruiz.
}

Servicio de Ginecología, Hospital Central Norte de Petróleos Mexicanos (PEMEX), México, DF.

\section{RESUMEN}

El avance de la tecnología y de las nuevas técnicas quirúrgicas en las especialidades médicas, ha convertido a la cirugía de mínima invasión ginecológica en una alternativa para el beneficio de los pacientes, obteniendo en la mayoría de los casos mejores resultados que la cirugía convencional. El objetivo de este documento es describir cuales son los mejores recursos y técnicas para el desarrollo y mantenimiento de destrezas en cirugía laparoscópica ginecológica, así como evidenciar los factores que favorecen o afectan la curva de aprendizaje y la calidad del desempeño. Así mismo, la metodología que supervisa el aprendizaje también puede examinar la competencia y la calidad del desempeño continuo en los procedimientos quirúrgicos. No existe una diferencia significativa entre las técnicas de entrenamiento en cirugía laparoscópica de mínima invasión, pero si es evidente que el entrenamiento previo mejora la habilidad individual y se obtienen resultados precisos y reproducibles. Es difícil mantener la consistencia, por lo que la ejecución deberá ser supervisada por métodos cuantitativos a fin de disminuir las complicaciones. Es evidente que se deben actualizar los programas académicos en la especialidad de ginecología, donde se incorpore un verdadero módulo de cirugía laparoscópica para que los médicos en formación adquieran destrezas con los procedimientos de baja complejidad, y al desarrollarse en los niveles de dificultad quirúrgica, se pueda supervisar la calidad del desempeño con métodos estadísticos adecuados.

\section{PALABRAS CLAVE: Cirugía laparoscópica ginecológica, curva de aprendizaje}

\section{SUMMARY}

The advancement of new surgical techniques in medical specialties has become minimally invasive gynecologic surgery into a modern surgical technique. There are a number of advantages to the patient with laparoscopic surgery versus an open procedure, obtaining in most cases mayor benefits than traditional surgery techniques. The purpose of this study was to describe which are the best aspects and techniques for developing and maintaining skills in gynecologic laparoscopic surgery and identify the factors affecting learning curves. A lot of factors are involved: like institutional policies and the characteristics of the surgeon such as attitude and capacity for acquiring new skills. Prior laparoscopic training has been shown to facilitate the process of learning, reduces complication rates and operative time. The type of training the surgeon has received is not significantly related to this learning curve. Maintaining consistency is challenging: however, assessing laparoscopic learning can also examine surgical competence. Consequently the implementation should be monitored by quantitative methods in order to provide both numerical and graphical representation of the learning process. The learning curves combined with the advantages of feedback using the rating 
scales open the possibility to design high-quality training curricula in advanced laparoscopy. It is clear that academic programs must be updated: this leads to an increasing demand for evidence and proficiencybased education, training and assessment of gynecologic laparoscopic skills. We need a feasible, structured and objective statistical system for assessment of both technical and procedural skills.

\section{KEY WORDS: Gynecologic laparoscopic surgery, learning curve}

\section{INTRODUCCIÓN}

El avance de la tecnología y de las nuevas técnicas quirúrgicas en las especialidades médicas, han convertido a la cirugía de mínima invasión ginecológica en una alternativa para el beneficio de los pacientes, obteniendo en la mayoría de los casos mejores resultados que la cirugía convencional (1-3).

Los programas académicos de residencias médicas en ginecología de todas las universidades del país no incluyen de manera formal la capacitación y adiestramiento para estas nuevas técnicas (1), por lo cual en 1998 se crearon en México los cursos de posgrado para médicos especialistas, hoy llamados "Cursos de Posgrado de Alta Especialidad", definidos como un conjunto de actividades académicas, prácticas asistenciales y de investigación clínica que debe cumplir un especialista que disponga de tiempo completo durante un año, sobre un campo muy circunscrito del conocimiento relacionado con su especialidad, a gran profundidad y actualidad $(1,4)$.

La adquisición de idoneidad es parte integral de los procedimientos quirúrgicos; por ello los programas de enseñanza han estandarizado sus criterios en tres niveles de entrenamiento en cirugía de mínima invasión (5) (Tabla I).
El objetivo de este documento es describir cuales son los recursos para el desarrollo y mantenimiento de habilidades en cirugía laparoscópica ginecológica, revelando los factores que afectan la calidad de la enseñanza. Así mismo, se evidencia que se pueden aplicar los mismos métodos estadísticos para vigilar la calidad de la curva de aprendizaje como para supervisar el desempeño de los médicos entrenados.

\section{MODELOS PARA EL ENTRENAMIENTO LAPA- ROSCÓPICO}

Las variedades de simuladores tanto animados como inanimados, tienen la finalidad de desarrollar destrezas que disminuyan el tiempo quirúrgico y las complicaciones, cuando se realicen los procedimientos en seres humanos $(2,5)$.

Las principales habilidades que se deben adquirir para avanzar en el desempeño de este procedimiento, es el cambio a una visión en dos dimensiones, una nueva coordinación óculo-manual en campo operatorio pequeño y acción de pivote de los instrumentos en la pared de los pacientes con instrumentos largos para amplificación de movimientos finos (6). Todos los problemas a solucionar pueden

Tabla I

NIVELES DE DIFICULTAD DE PROCEDIMIENTOS ENDOSCÓPICOS DE ACUERDO AL RCOG (5)

\begin{tabular}{lll}
\hline Nivel 1 & Nivel 2 & Nivel 3 \\
\hline laparoscopia diagnóstica & adherensiolisis menor & miomectomía \\
esterilización tubaria & embarazo ectópico & adherensiolisis profunda asociada \\
prueba de permeabilidad tubaria & endometriosis leve & a patología \\
& cistectomía & histerectomía total laparoscópica \\
& salpingo-oforectomía & endometriosis \\
& salpingotomía & (estadio iii/iv) \\
& histerectomía laparoscópica & procedimientos de incontinencia \\
& asistida vía vaginal sin patología & procedimientos de suspensión \\
& asociada & \\
& histerectomía subtotal sin patología & \\
& asociada & \\
\hline
\end{tabular}


minimizarse con el uso continuo de pelvitrainer y simuladores virtuales, donde se pueden repetir los movimientos y ejercicios cuantas veces sea necesario hasta dominar la técnica a bajo costo y sin causar lesiones en animales vivos o pacientes $(6,7)$.

A continuación se describe el orden sugerido para que el personal médico en formación, logre disminuir la curva de aprendizaje o bien para que los entrenados mantengan un entrenamiento satisfactorio (6-8).

\section{Los simuladores virtuales y el pelvitrainer}

Tienen la finalidad que el alumno adquiera o conserve el dominio del instrumental laparoscópico y ejercite movimientos quirúrgicos (disección, corte, sutura y anudado), habituándose al uso de ambas manos y coordinándose entre ellas $(6,9)$.

Los simuladores virtuales de realidad son juegos de ordenador: compuestos por un bastidor estándar, instrumentos laparoscópicos y un pedal digital conectados electrónicamente a un ordenador personal $(9,10)$. El software construye un entorno virtual visto en la pantalla de la computadora, que muestra la posición y movimiento de los instrumentos laparoscópicos en tiempo real dentro de la cavidad abdominal $(7,10)$.

A medida que el participante realiza una tarea, se registra en el sistema cada movimiento y error, determina el tiempo y la eficacia de movimientos con cada mano para el análisis objetivo (11). Los programas varían considerablemente: baja fidelidad o simple tarea orientada a objetos de simulación y alta resolución con gráficos en tres dimensiones, que crea un virtual precisión anatómica (12) (Figura 1).
Los 5 componentes fundamentales en la realidad virtual son los siguientes: fidelidad (¿parece real?), propiedades del objeto (¿los órganos se deforman con la presión o la caída con la gravedad?), interactividad (¿la interacción entre los instrumentos y el órgano es realista?), información sensorial (¿hay retroalimentación de fuerza?) y reactividad (¿los órganos deben reaccionar de forma apropiada con sangrado?) $(11,12)$.

Los pelvitrainer utilizan equipo real de cirugía laparoscópica incluyendo: laparoscopio, cámara, fuente de luz, trocares e instrumentos laparoscópicos que se colocan a través de una cubierta opaca sobre el área de formación $(6,7)$. Este método de evaluación y retroalimentación puede estar lleno de prejuicios y escasa fiabilidad por la relación entre el participante y el experto (7) (Figura 2). Cuenta con las siguientes ventajas:

a) La adquisición de habilidades específicas.

b) La posibilidad de prácticas repetidas y de bajo costo para evaluar el desempeño.

c) Entrenamiento sin riesgos y en un ambiente relajado, practicando a solas o con el operador del laparoscopio habitual, sin tener que estar en un lugar especialmente diseñado para ello (8).

d) Permite perfeccionar los procedimientos difíciles (9).

Las mejoras en estas propiedades se asocian con un número mayor de costos para el sistema de formación $(11,12)$. Así mismo, existe una escala de clasificación mundial que ha demostrado una alta fiabilidad y validez en la evaluación del participante, que ha evidenciado habilidades uniformes en cirugía de mínima invasión, y es capaz de detectar si el operador se encuentra intoxicado o presenta falta de sueño (los

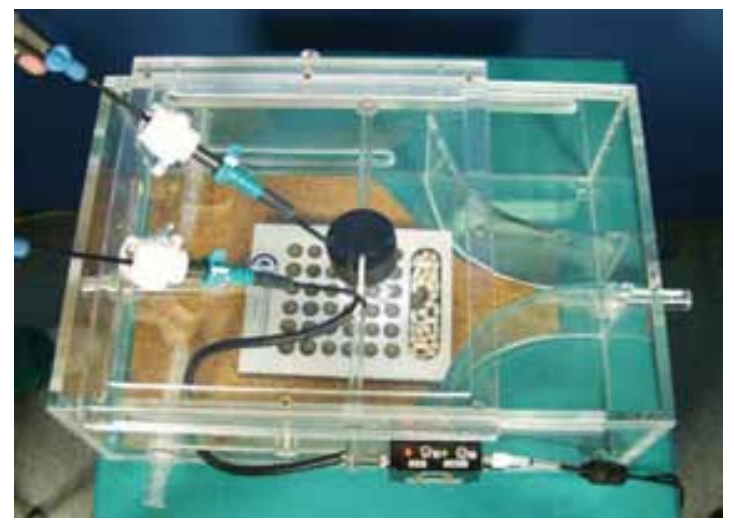

Figura 1. Entrenamiento en pelvitrainer.

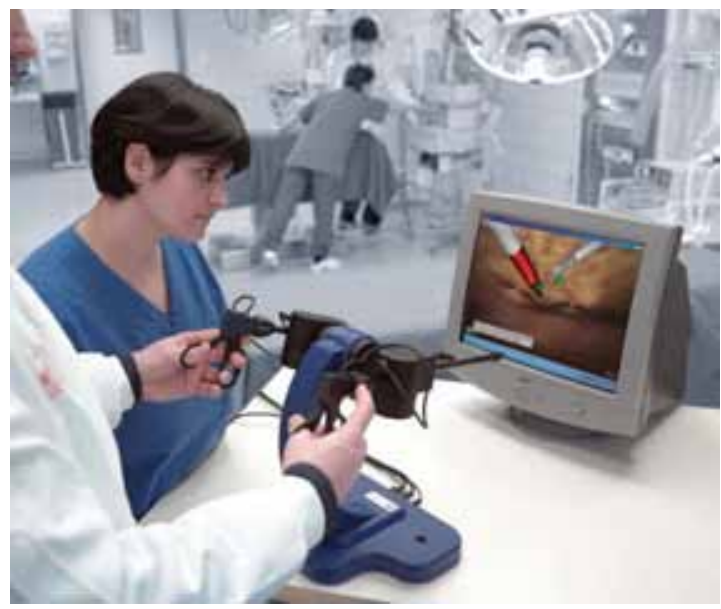

Figura 2. Entrenamiento en simulador virtual. 
cirujanos bien descansados y en óptimas condiciones tienen menos errores que si presentan alguna alteración); se trata de Estructuras de Evaluación Técnica de Habilidades (OSATS) y es el estándar para la evaluación de competencias (7,13-15).

En general, estos recursos didácticos permiten modificar el viejo modelo de enseñanza "tipo aprendices", hacia una instrucción de habilidades con un método lógico y sistemático, haciendo más que observando (10).

\section{Modelos animales vivos}

Los modelos animales vivos son comparables en anatomía y se ha establecido su uso para el entrenamiento quirúrgico. Sin embargo, los gastos de manutención y del bioterio en general son muy caros, además de la complejidad técnica del cuidados de los animales y de las consideraciones éticas establecidas son importantes para el uso de estos, por lo que no son opciones óptimas para las repetitivas prácticas y tareas necesarias en la formación del personal en entrenamiento $(7,8)$ (Figura 3).

Las prácticas en animales es donde se sugiere realizar procedimientos complejos siguiendo los mismos pasos como se haría en el paciente vivo $(8,13)$.

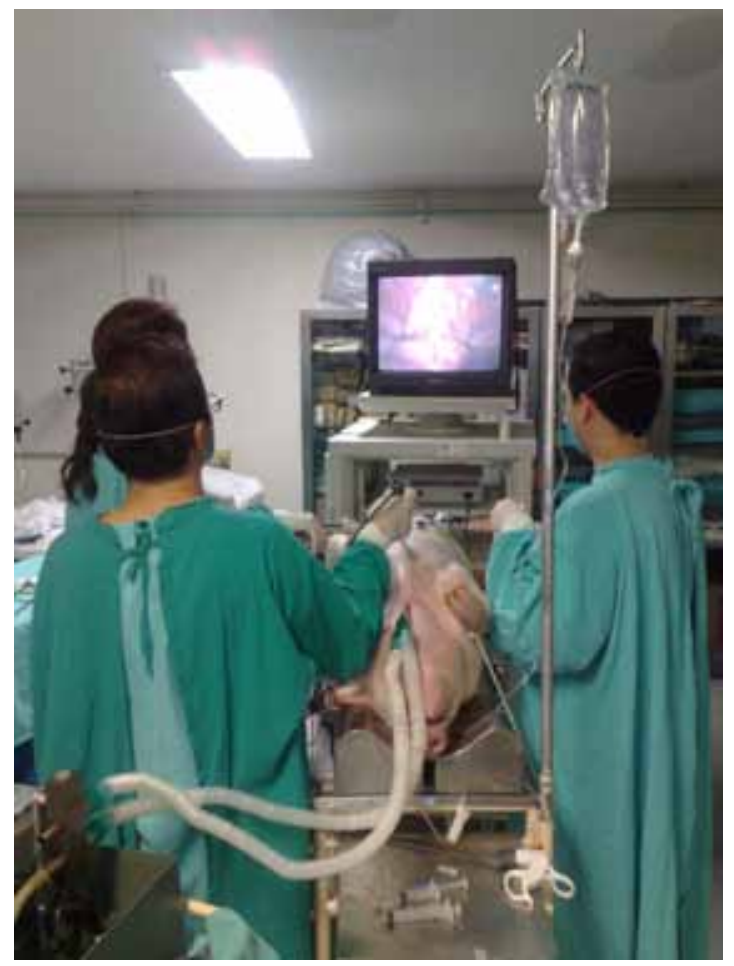

Figura 3. Entrenamiento en animales

\section{Demostraciones prácticas con expertos}

Las demostraciones prácticas desarrolladas por ginecólogos expertos, tienen la finalidad de observar detalles técnicos, así como la solución de complicaciones que surgen en el momento del procedimiento $(7,14,15)$.

El entrenamiento con expertos en cadáveres tiene la ventaja de tener la anatomía exacta y un gran realismo, pero son caros, escasos y en ellos se ha alterado la consistencia de los tejidos $(7,8,15)$.

\section{Enseñanza por medios audiovisuales}

La enseñanza por medios audiovisuales (videos), sirve para observar el mismo procedimiento pero realizado por diferentes cirujanos para ver la alternativas a la técnica ya estudiada $(8,10)$.

\section{Verificación de la competencia inicial}

La supervisión por un médico experto en las primeras cirugías realizadas en seres humanos, tiene la finalidad de evitar complicaciones (7).

La complejidad de los diferentes tipos de cirugía pueden influir también en la curva de aprendizaje, conforme un cirujano adquiere más experiencia, puede realizar continuamente procedimientos difíciles, siendo este el factor importante para valorar si se ha llegado al punto óptimo en dicha curva (1416). Sin embargo, es conocido que los ginecólogos en hospitales de enseñanza continúan con su propia curva de aprendizaje, lo que traduce falta de oportunidades para que el residente participe como cirujano principal, provocando como consecuencia una falta de práctica y habilidad en cirugía laparoscópica $(15,16)$. De ahí la importancia de unificar y sistematizar la supervisión de calidad en el aprendizaje y en el desempeño habitual $(17,18)$.

\section{DISCUSIÓN}

La capacidad para realizar con seguridad un procedimiento laparoscópico puede disminuirse o aumentarse durante la curva de aprendizaje por numerosos factores, como son la aptitud quirúrgica, la destreza manual, el conocimiento de la anatomía, el centro de formación, el número de pacientes, la presencia de estandarización de la técnica quirúrgica y la supervisión del experto (16).

Ante ello, se ha evidenciado que el inicio del entrenamiento en cirugía de mínima invasión usando pelvitrainer, modelos virtuales y animales vivos, mejora las habilidades quirúrgicas al momento de operar en seres humanos. Con ello disminuye el 
tiempo quirúrgico y se reducen las complicaciones (19): los cirujanos sin experiencia en este tipo de técnicas tienen tasas más altas de complicaciones operatorias por procedimiento (20), aunque cabe señalar que no se ha establecido la equivalencia en horas de entrenamiento con simuladores virtuales o pelvitrainer a cirugía en vivo $(21,22)$. La literatura mundial ha confirmado que las habilidades quirúrgicas endoscópicas son mejores en cirujanos que previamente hubieran jugado video juegos (principalmente los de deportes) y aquellos que utilizan instrumentos musicales (23). También se ha referido que no existe diferencia estadísticamente significativa entre hombres y mujeres residentes que utilizaron simuladores de realidad virtual en términos de error o de la economía del movimiento (24).

Las fallas y la falta del equipo laparoscópico son una de las causas que comúnmente alteran la curva de aprendizaje. Lo evidencian Courdier y cols (25), quienes realizaron un estudio donde se dividió el mal funcionamiento del equipo de laparoscopia en 4 rubros (Tabla II): del total de procedimientos con deficiencias el error humano afectó el $47,4 \%$ (distribuidos en responsabilidad de la enfermera $72 \%$, técnico biomédico $16 \%$ y el cirujano $12 \%$ ), la falla del equipo laparoscópico el $42,3 \%$ y el $10,3 \%$ fue debido al personal no entrenado en el uso del material (25). Esto ultimo revela que los equipos de laparoscopia son cada vez más sofisticados: es necesaria la capacitación especial para su manejo y con ello disminuir errores en la aplicación y tiempo de armado, situación que afecta el tiempo quirúrgico del procedimiento (25).

Se propone que el cirujano realice una lista de verificación para el instrumental (a fin de disminuir tiempos muertos por falta de equipo o error en las conexiones del mismo), así como examinar su funcionamiento previo al evento con el objetivo de brindar seguridad al paciente y lograr el curso ade-

\section{Tabla II}

\section{FALLAS MÁS FRECUENTES DEL EQUIPO LAPAROSCÓPICO}

\begin{tabular}{lc}
\hline Tipo de fallas & $\%$ de falla \\
\hline $\begin{array}{l}\text { 1. Imágenes: cabeza cámara, cables } \\
\text { de video, pantalla y sus conexiones }\end{array}$ & 12,1 \\
$\begin{array}{l}\text { 2. Transmisión entre } 2 \text { elementos: } \\
\text { líquido, gas y luz, succión y sistema } \\
\text { de irrigación }\end{array}$ & 36,2 \\
$\begin{array}{l}\text { 3. Circuito eléctrico: generador, ca- } \\
\text { bles, pinza bipolares, corte monopolar }\end{array}$ & 22,4 \\
4. Instrumentos quirúrgicos & 29,3 \\
\hline
\end{tabular}

cuado de la intervención, concluyendo que lo más importante es identificar los problemas para establecer estrategias que optimicen el funcionamiento de los equipos de laparoscopia (25).

Aunque existen algunos reportes donde nos informan cuantos procedimientos laparoscópicos requiere un cirujano en adiestramiento para disminuir la morbilidad de las pacientes, no se han presentado los resultados del proceso de forma consecutiva evaluando la competencia de un número de examinadores expertos en un período determinado de tiempo, considerando los errores sistemáticos y aleatorios (16-18).

Para ello, se ha sugerido el control de calidad basado en un análisis de gráficos CUSUM (cumulative sums method) que son una representación de la tendencia y el desenlace al medir una serie de procedimientos $(15,16,26,27)$. Las pruebas secuenciales de este tipo tienen algunas ventajas cuando se comparan con los métodos tradicionales de análisis de resultados: la independencia del tamaño muestral, una mayor potencia para detectar cambios transitorios en las tendencias, la continuidad del análisis en el tiempo y la posibilidad de realizar una evaluación rápida de los datos $(17,26,27)$. Por lo anterior, las curvas de aprendizaje CUSUM, no solo sirven como indicador del rendimiento satisfactorio para adquirir la habilidad clínica, sino también para evaluar la calidad del desempeño y como una medición continua de los efectos que pudieran provocar cualquier cambio en la técnica $(18,27)$.

Así mismo, en los reportes previos de curvas de aprendizaje, tampoco existe información del fallo aceptable e inaceptable durante un procedimiento supervisado, por lo que es evidente que la información que se genere deberá estar basada en un método de control de calidad que revele el desempeño a través del tiempo y suministre una evidencia objetiva basada en un análisis caso por caso $(2,4,16,18,26)$.

Es oportuno detallar la relevancia en el hecho de que la metodología que verifica la aptitud para la enseñanza, también puede examinar la competencia y la calidad de los procedimientos quirúrgicos: el constatar la idoneidad cobra cada día mayor interés en los centros especializados por el gran impacto que suponen para las instituciones: a) el exceso en horas-operador empleados en los centros de atención hospitalaria que no cuentan con personal capacitado o con la competencia requerida para la realización de un procedimiento, b) el exceso de recursos empleados en la realización de procedimientos complejos y avanzados cuando ocurren complicaciones y c) la alta prevalencia de conflictos legales asociados a mala praxis derivada de 
la aplicación de procedimientos con fallas, lo que ha obligado al gremio médico y a las instituciones prestadoras de servicios de salud a implementar controles de calidad efectivos (15-18,27).

Dadas las condiciones referidas, se ha sugerido que el asesoramiento de calidad en las técnicas laparoscópicas debe incluir revisiones y auditorias regulares al desempeño de los equipos (aspecto técnico) y también a los parámetros relacionados con los operadores, pues queda claro que ambos componentes del procedimiento laparoscópico ginecológico no deben disociarse (17).

A pesar de los múltiples dilemas, es evidente que la falta de formación estructurada durante la residencia, podría ser el principal factor limitante en la aplicación de tecnologías avanzadas a la cirugía laparoscópica en la práctica habitual $(14,15,19)$. Chiasson y cols (21), reportan que solo el $18 \%$ de los residentes de cirugía se encuentran preparados para realizar procedimientos laparoscópicos: la contratación de un experto laparoscopista en realizar procedimientos avanzados, ha demostrado ser una manera segura y eficaz para enseñar habilidades y procedimientos, e iniciar la supervisión estadística de la curva de aprendizaje y el desempeño de los médicos residentes (28).

\section{CONCLUSIONES}

Se ha demostrado que sin importar el tipo de simulador, el usuario siempre mejora las habilidades entrenadas (7). No existe una diferencia significativa para saber cuál es el mejor entrenamiento previo a realizar procedimientos de mínima invasión, pero si está demostrado que el entrenamiento previo mejora por mucho la habilidad quirúrgica, optimiza los tiempos quirúrgicos, disminuye las complicaciones y acorta la curva de aprendizaje, en comparación con el personal que utiliza el método de enseñanza antiguo en la cirugía abierta de ver y hacer en el paciente $(7,8,11)$.

Como es posible observar, se requieren niveles de conocimiento y de entrenamiento adecuados, por lo que es necesario conocer el número mínimo de casos que se requieren en cada población para que un operador realice procedimientos confiables. Esta información podría generarse con el recurso de las gráficas tipo CUSUM que son una representación de la tendencia y el desenlace, al medir una serie de procedimientos (26-27). Las pruebas secuenciales de este tipo tienen múltiples ventajas, pues sirven como indicador del rendimiento satisfactorio para adquirir la habilidad quirúrgica y también evalúan la calidad del desempeño, al medir continuamente los efectos que pudieran provocar cualquier cambio en la técnica $(18,27)$.

Es tiempo de actualizar los programas académicos en la especialidad donde se incluya un verdadero módulo de cirugía laparoscópica ginecológica, con el fin de que los médicos en formación adquieran destrezas estandarizadas para la ejecución de procedimientos de baja complejidad, facilitando también el avance de nivel laparoscópico, se acorte el tiempo quirúrgico y se disminuyan las complicaciones si se desarrolla un posgrado.

\section{REFERENCIAS}

1. Morales Castillo ME, Ruiz Pérez LC. Cursos de posgrado para médicos especialistas. Rev Fac Med UNAM 2006;49(1). Hallado en: http://www.ejournal. unam.mx/rfm/no49-1/RFM49107.pdf

2. Latiff $A$. La curva de aprendizaje en la cirugía: ¿qué es?, ¿cómo se mide? Rev Col Uro;2005:15-7.

3. Michel LA. The epistemology of evidence-based medicine. Surg Endosc 2007;21:145-51.

4. Cook JA, Ramsay CR, Fayers P. Statistical evaluation of learning curve effects in surgical trials. Clinical Trial 2004;1:421-7.

5. Royal College of Obstetricians and Gynaecologists. Classification of laparoscopic procedures per level of difficulty. RCOG, London, 2001.

6. Justo-Janeiro JM, Pedroza-Meléndez A, Prado E, Theurel-Vincent G, Vázquez-de Lara L. Un nuevo simulador en laparoscopia. Cir Ciruj 2007;75:19-23.

7. Chou B, Handa VL. Simulators and virtual reality in surgical education. Obstet Gynecol Clin North Am 2006;33:283-96.

8. Vázquez-Ortega $L$, Shuck-Bello $C$, Cantellano-Orozco $\mathrm{M}$, Camarena-Reynoso H, Leos-Acosta C, AndradePlatas D, et al. Curva de aprendizaje en cirugía laparoscópica: experiencia de nefrectomía laparoscópica en bioterio. Rev Mex Urol 2008;68:166-9.

9. Munz Y, Kumar BD, Moorthy K, Bann S, Darzi A. Laparoscopic virtual reality and box trainers: is one superior to the other? Surg Endosc 2004;18:485-94.

10. Uchal M, Raftopoulos Y, Tjugum J, Bergamaschi R. Validation of a six task simulation model in minimally invasive surgery. Surg Endosc 2005;19:109-16.

11. Youngblood PL, Srivastava $S$, Curet $M$, Heinrichs WL, Dev P, Wren SM. Comparison of training on two laparoscopic simulators and assessment of skills transfer to surgical performance. J Am Coll Surg 2005;200:546-51.

12. Kothari S, Kaplan B, DeMaria E, Broderick TJ, Merrell $\mathrm{RC}$. Training in laparoscopic suturing skills using a new computer-based virtual reality simulator (MISTVR) provides results comparable to those with an established pelvic trainer system. J Laparoendosc Adv Surg Tech A 2002;12:167-73.

13. Schijven MP, Berlage JT, Jakimowicz JJ. Minimalaccess surgery training in the Netherlands: a survey among residents-in-training for general surgery. Surg Endosc 2004;18:1805-14. 
14. Jansen FW, Kolkman W. Implementation difficulties of advanced techniques in gynecological laparoscopy. Gynecol Surg 2008;5:261-4.

15. Van Hove PD, Tuijthof GJ, Verdaasdonk EG, Stassen LP, Dankelman J. Objective assessment of technical surgical skills. Br J Surg 2010;97:972-87.

16. Traxer O, Gettman MT, Napper CA, Scott DJ, Jones DB, Roehrborn CG, et al. The impact of intense laparoscopic skills training on the operative performance of urology residents J Urol 2001;166:1658-61.

17. Cruz-Martinez R, Figueras F, Moreno-Alvarez $O$, Martinez JM, Gomez O, Hernandez-Andrade E, Gratacos E. Learning curve for lung area to head circumference ratio measurement in fetuses with congenital diaphragmatic hernia. Ultrasound Obstet Gynecol 2010;36:32-6.

18. Ville Y. "Ceci nest pas une échographie": a plea for quality assessment. Ultrasound Obstet Gynecol 2008;31:1-5.

19. Rosser JC Jr, Lynch PJ, Cuddihy L, Gentile DA, Klonsky J, Merrell R. The impact of video games on training surgeons in the 21 st Century. Arch Surg 2007;142:181-6.

20. Seymour NE, Gallagher AG, Roman SA, O'Brien MK, Bansal VK, Andersen DK, Satava RM. Virtual reality training improves operating room performance: results of a randomized, double-blinded study. Ann Surg 2002;236:458-64.

21. Chiasson PM, Pace DE, Schlachta CM, Mamazza J,
Poulin EC. Minimally invasive surgery training in Canada: a survey of general surgery residents. Surg Endosc 2003;17:371-7.

22. Munz Y, Kumar BD, Moorthy K, Bann S, Darzi A. Laparoscopic virtual reality and box trainers: is one superior to the other? Surg Endosc 2004;18:485-94.

23. Harper JD, Kaiser S, Ebrahimi K, Lamberton GR, Hadley HR, Ruckle HC, Baldwin DD. Prior video game exposure does not enhance robotic surgical performance. J Endourol 2007;21:1207-10.

24. Brummitt K, Harmanli OH, Gaughan J, Dandolu V, Chatwani AJ, Hernandez E. Gynecologists' attitudes toward hysterectomy: is the sex of the clinician a factor? J Reprod Med. 2006;51:21-5.

25. Courdier S, Garbin O, Hummel M, Thoma V, Ball E, Favre R, Wattiez A. Equipment failure: causes and consequences in endoscopic gynecologic surgery. J Minim Invasive Gynecol 2009;16:28-33.

26. Biau DJ, Porcher R, Salomon JL. CUSUM: a tool for ongoing assessment of performance. Ultrasound Obstet Gynecol 2008;31:252-5.

27. Bolsin S, Colson M. The use of the cusum technique in the assessment of trainee competence in new procedures. Int J Qual Health Care 2000;12:433-8.

28. Kolkman W, Engels LE, Smeets MJ, Jansen FW. Teach the teachers: an observational study on mentor traineeship in gynecological laparoscopic surgery. Gynecol Obstet Invest 2007;64:1-7. 